\title{
Gradientes, estabilidad y estado de conservación de peces en la cuenca alta del río Turbio, vertiente andina del Orinoco, Venezuela
}

\author{
Douglas Rodríguez-Olarte \& Margenny Barrios Gómez \\ Colección Regional de Peces. Museo de Ciencias Naturales UCLA, Departamento de Ciencias Biológicas, Decanato \\ de Agronomía, Universidad Centroccidental Lisandro Alvarado, UCLA. Apartado postal 400, Barquisimeto, Estado \\ Lara, Venezuela; douglasrodriguez@ucla.edu.ve,margennybarrios@gmail.com
}

Recibido 14-X-2013. Corregido 28-I-2014. Aceptado 27-II-2014.

\begin{abstract}
Gradients, stability and conservation status of fishes in the Andean mountain streams of the Orinoco versant, Venezuela. Most Andean mountain streams of the Orinoco Basin in Venezuela, had been suffering various disturbances. In spite of this, fish communities have been poorly evaluated in terms of spatial and temporal gradients. Thus, the generation of information about these communities is a priority, since it may support conservation of regional hydrobiological resources. For this, six sites were monthly evaluated in tributaries of upper Turbio River in Sierra de Portuguesa (770-1 305msnm), during the dry season and the beginning of the rainy season (January-April 2012); we characterized stream dimensions, substrate and water physicochemical variables. Electrofishing was used to determine the number and abundance of fish species. The fish communities and their stability were evaluated by exploring the changes in richness and abundance, coefficients of variation, hierarchical classification and non-metrical multidimensional scaling analysis. The sites conservation status was estimated with a habitat integrity index. Our results showed that richness of the 12 species found varied according to the mountain elevation, the tributaries and the conservation status of the sites. Chaetostoma dorsale, Creagrutus taphorni and Chaetostoma milesi comprised $90.8 \%$ of the relative abundance, and maintained the first ranks during the study period. The communities had little spatio-temporal variation which was associated with a gradient signed principally by the amount of shadow, rocks and gravel cover, water temperature and conductivity. Stability was moderate but varied with elevation and according to site conservation status. The tributaries in upper Turbio River were found to be under severe impacts and their fishes were found impoverished and at risk. Rev. Biol. Trop. 62 (3): 987-996. Epub 2014 September 01.
\end{abstract}

Key words: fish communities, environmental gradients, Andean streams, hydrobiological resources, Venezuela.

El estudio de los patrones espaciales y temporales en los peces de las montañas andinas al norte de Suramérica ha sido poco estudiado en relación con las prospecciones sobre la diversidad biológica regional, aun cuando estas ictiofaunas son diversas, con elevado endemismo y se encuentran en riesgo en algunos drenajes (Anderson \& Maldonado-Ocampo, 2011). En estos ríos de montaña al disminuir la altura aumentan la riqueza, composición de especies y gremios (Jaramillo-Villa, MaldonadoOcampo, \& Escobar, 2010), mientras que en los cauces disminuyen el tamaño de las granulometrías y la velocidad de la corriente, pero se incrementan las concentraciones de sólidos en las aguas (Lujan et al., 2013). La menor variación ambiental en los ríos de montaña sugiere una mayor estabilidad de las comunidades de peces a largo plazo (Grossman, Dowd, \& Crawford, 1990), lo que puede estar asociado con hábitats menos complejos y poco cambiantes en el tiempo. La estabilidad varía con la altura en arroyos del piedemonte andino del Orinoco, donde los cambios temporales en las comunidades de peces son predecibles y estas pueden ser más estables respecto a aquellas en los llanos altos a menor altitud (Winemiller, Agostinho, \& Pellegrini-Caramaschi, 2008), 
pero este gradiente no es conocido a mayores alturas en la región, donde existen notorios gradientes climáticos y antropogénicos.

La estabilidad de las comunidades de peces obedece también al estado de conservación de los ríos, pues aquellos que son perturbados, ya sea en su granulometría, aguas o por la fragmentación en sus bosques ribereños, generalmente tienen comunidades de peces simplificadas $\mathrm{y} / \mathrm{o}$ con baja integridad respecto a condiciones ideales (Allan, 2004). En piedemontes andinos de Venezuela, por ejemplo, el deterioro del hábitat fluvial y el ambiente ribereño predicen una simplificación en la estructura de las comunidades de peces (Rodríguez-Olarte, Coronel, Taphorn, \& Amaro, 2006); sin embargo, se desconoce cómo varían estas y su hábitat en el tiempo y a mayores alturas, principalmente en drenajes sujetos a perturbaciones intensas.

El estado de conservación de los ríos de montaña en la vertiente andina del Orinoco ha sido poco evaluado, pero en algunos casos, como en la cuenca alta del río Turbio, es evidente una depauperación en el funcionamiento y permanencia de sus tributarios, lo que debería reflejarse en los atributos de sus ictiofaunas (riqueza, abundancia y estabilidad, por ejemplo), pero se desconoce el estado de conservación de estos ríos y de los patrones espaciales y temporales de sus comunidades de peces. Este vacío de información limita la potencial elaboración y aplicación de programas de manejo de los recursos hidrobiológicos locales. De acuerdo con lo anterior se evaluaron los tributarios y sus comunidades de peces durante el periodo de sequía y principios del periodo de lluvias, con los objetivos específicos de (1) identificar patrones espaciales y temporales en la organización y estabilidad de las comunidades de peces, y (2) relacionar los atributos de tales comunidades con las características del hábitat fluvial y el estado de conservación de los ríos.

\section{MATERIALES Y MÉTODOS}

Área de estudio: La cuenca alta del río Turbio está conformada por los tributarios Río
Claro y Turbio, y drena las estribaciones de la vertiente nororiental de los Andes en la Sierra de Portuguesa, y agrupa los drenajes desde las mayores alturas $\left(2165\right.$ m.s.n.m. $10^{\circ} 02 ' 55^{\prime \prime} \mathrm{N}$ $69^{\circ} 17^{\prime} 09$ ' W) hasta la desembocadura del Río Claro en el río Turbio (465m.s.n.m. 0943'42" $\mathrm{N}-69^{\circ} 35^{\prime} 46^{\prime \prime} \mathrm{W}$ ), donde predominan valles intramontanos con pendientes moderadas, enmarcados en geomorfologías accidentadas. En Río Claro la precipitación es unimodal con un promedio anual de $1200 \mathrm{~mm}(945 \mathrm{msnm})$ y el periodo de lluvias es desde abril a noviembre; mientras que en las cabeceras del Turbio (1 $902 \mathrm{msnm}$ ) el régimen es bimodal, con $964 \mathrm{~mm}$ anuales y con dos máximos pluviométricos en abril-junio y octubre (López \& Andressen, 1996). El periodo de sequía (en adelante llamado sequía) es desde enero a marzo. La vegetación es principalmente secundaria, con bosques húmedos $\mathrm{y}$ densos en las cumbres $\mathrm{y}$ con bosques ralos y matorrales xerófilos con menor cobertura del suelo en el resto de la cuenca (Smith, 1991). Sobre los 1 000ms.n.m. se ubican los parques nacionales Terepaima y Yacambú, pero allende a los mismos la deforestación, los incendios y la agricultura son eje transversal en el paisaje, y en los cauces es habitual la minería no metálica y el ingreso de aguas servidas sin tratamiento provenientes de los centros urbanos, complejos industriales y sistemas de producción agropecuaria.

Muestreos y análisis de datos: En los tributarios Río Claro (RC1, RC2), Guayamure (un afluente de Río Claro, RG1) y Turbio (RT1, RT2, RT3) se ubicaron seis localidades que fueron muestreadas mensualmente $(\mathrm{n}=24)$ en sequía (enero, febrero y marzo) y a principio de las lluvias (abril) de 2012. En cada localidad (longitud 50m) se midieron el ancho húmedo y la profundidad del río con cintas métricas, y la velocidad de la corriente mediante la relación entre el tiempo y la distancia recorrida en el cauce por un objeto flotante. En tres transectos perpendiculares al cauce se estimó, en unidades de $1 \mathrm{~m}^{2}$, la cobertura porcentual de los componentes del sustrato (fango, arena, gravas, cantos rodados, piedras, rocas, ramas y hojas) 
y de la sombra aportada por la vegetación ribereña (Barbour, Gerritsen, Snyder, \& Stribling, 1999). Con sensores portátiles se midió la conductividad $(\mu S / \mathrm{cm})$, los sólidos totales disueltos $(\mathrm{mg} / \mathrm{L})$, el pH y la temperatura $\left({ }^{\circ} \mathrm{C}\right)$ (Cuadro 1$)$. Se empleó la electropesca con remoción única (Rodríguez-Olarte et al., 2006) y sin límite de tiempo. Los especímenes fueron identificados, contados y devueltos vivos a su hábitat, pero algunos fueron incorporados a la Colección Regional de Peces (CPUCLA, Cuadro 2) del Museo de Ciencias Naturales de la UCLA. La validez en la identificación de las especies fue actualizada (Froese \& Pauly, 2013).
La mayoría de las variables no tuvieron distribución normal (prueba de Shapiro-Wilk, $\mathrm{p} \leq 0.05$ ). Se transformaron las coberturas porcentuales del sustrato mediante la raíz cuadrada del arcoseno, mientras que el ancho, profundidad y sólidos disueltos totales lo fueron con $\log (\mathrm{x})$. Para identificar la importancia de cada especie se emplearon rangos de abundancia. Para reconocer efectos significativos en los atributos de las comunidades y las variables ambientales en y entre las localidades, así como en el tiempo, se emplearon las pruebas no paramétricas de rangos signados de Wilcoxon, $\mathrm{U}$ de Mann-Whitney y Kruskal-Wallis (Quinn \&

\section{CUADRO 1}

Variables ambientales en grupos de localidades detectados en los análisis multivariados y sus correlaciones $\left(\mathrm{r}_{\mathrm{s}}\right)$ respecto al eje NMS1. Los valores son promedios (entre paréntesis los mínimos y máximos)

TABLE 1

Environmental variables in groups of sites detected in multivariate analysis and their correlations $\left(\mathrm{r}_{\mathrm{s}}\right)$ to the axis NMS1. Values are mean (in brackets minimum and maximum)

\begin{tabular}{|c|c|c|c|c|}
\hline \multirow{3}{*}{ Variables ambientales } & \multicolumn{3}{|c|}{ Localidades } & \multirow{2}{*}{$\mathrm{r}_{\mathrm{s}} \mathrm{NMS1}$} \\
\hline & Grupo a & Grupo b & Grupo c & \\
\hline & RC1-RG1-RT1 & RT2-RT3 & $\mathrm{RC} 2$ & $\mathrm{P}$ \\
\hline \multicolumn{5}{|l|}{ Cauces: } \\
\hline Altura (msnm) & 1 130.7(982-1305) & $835(770-900)$ & 962 & \\
\hline Ancho (m) & $3.82(1.5-5.3)$ & $5.42(3-9)$ & $3.66(3-4.7)$ & $-0.41 *$ \\
\hline Profundidad (cm) & $18.88(11.4-25.4)$ & $16.88(3-23.3)$ & $17.03(11.1-23.8)$ & -0.06 \\
\hline Velocidad (m/s) & $0.69(0.3-1.3)$ & $0.99(0.6-1.4)$ & $0.63(0.5-0.7)$ & $-0.41 *$ \\
\hline \multicolumn{5}{|l|}{ Coberturas (\%): } \\
\hline Fango & $0.84(0-5.8)$ & $1.61(0-6.3)$ & $4.08(0-9.4)$ & -0.20 \\
\hline Arena & $7.51(1.2-20.6)$ & $4.89(0-12.8)$ & $4.50(2.1-9.4)$ & 0.35 \\
\hline Grava fina & $14.68(8.5-32.3)$ & $21.73(10.7-52.8)$ & $16.62(11.4-25.3)$ & -0.37 \\
\hline Grava gruesa & $19.34(12.3-33.5)$ & $28.36(16.9-39)$ & $22.44(17.0-28.3)$ & $-0.58 * *$ \\
\hline Cantos rodados & $18.69(10.2-33.3)$ & $23.12(4.1-38.0)$ & $20.14(16.2-24.8)$ & $-0.47 *$ \\
\hline Piedras & $13.21(6.4-20.6)$ & $10.05(0-19.1)$ & $13.22(6.9-16.1)$ & 0.22 \\
\hline Rocas & $19.61(1.7-35.5)$ & $7.64(0-15.6)$ & $15.51(9.7-20.1)$ & $0.55 * *$ \\
\hline Ramas y hojas & $6.13(0.61-14.7)$ & $2.59(0-7.7)$ & $3.51(2.2-4.5)$ & $0.63 * *$ \\
\hline Sombra & $21.52(0.7-43.8)$ & $9.87(0-30)$ & $10.31(0.6-20.4)$ & $0.71 * *$ \\
\hline \multicolumn{5}{|l|}{ Aguas: } \\
\hline Temperatura $\left({ }^{\circ} \mathrm{C}\right)$ & $19.91(18.1-22)$ & $23(18.1-28.1)$ & $24.38(21.5-27.8)$ & $-0.74 * *$ \\
\hline Sólidos disueltos (mg/L) & $201(145-276)$ & $327.8(194-474)$ & $202(196-211)$ & $-0.60 * *$ \\
\hline Conductividad $(\mu \mathrm{S} / \mathrm{cm})$ & $417.9(280-598)$ & $685.3(397-1010)$ & $424.8(414-436)$ & $-0.62 * *$ \\
\hline $\mathrm{pH}$ & $8.17(7.1-8.7)$ & $8.24(8.2-8.6)$ & $8.31(8.1-8.5)$ & -0.01 \\
\hline Integridad del Hábitat (HII): & $79(58-96)$ & $39(34-43)$ & 40 & $0.95^{* *}$ \\
\hline
\end{tabular}

$(\mathrm{p}<0.05=*, \mathrm{p}<0.01=* *)$. 
CUADRO 2

Especies, abundancias (rangos entre paréntesis) y su correlaciones $\left(\mathrm{r}_{\mathrm{s}}\right)$ con el eje NMS1

TABLE 2

Species, abundances (ranks in parentheses) and their correlations $\left(\mathrm{r}_{\mathrm{s}}\right)$ to the axis NMS1

\begin{tabular}{|c|c|c|c|c|c|c|c|c|c|c|}
\hline \multirow{2}{*}{ Familia } & \multirow{2}{*}{ Especies } & \multicolumn{4}{|c|}{ Río Claro } & \multicolumn{4}{|c|}{ Turbio } & \multirow{2}{*}{$\begin{array}{c}\mathrm{r}_{\mathrm{s}} \\
\mathrm{NMS1} \\
\mathrm{P}\end{array}$} \\
\hline & & Ene & Feb & Mar & Abr & Ene & Feb & Mar & Abr & \\
\hline Characidae & 1. Bryconamericus cismontanus & 0 & 0 & 0 & 0 & $18(5)$ & $9(6)$ & $8(5)$ & $9(5)$ & $-0.73 * *$ \\
\hline Characidae & 2. Creagrutus taphorni & $218(2)$ & $107(2)$ & $143(2)$ & $118(2)$ & $54(3)$ & $157(1)$ & $129(2)$ & $74(1)$ & $0.72 * *$ \\
\hline Lebiasinidae & 3. Lebiasina erythrinoides & $7(4)$ & $3(4)$ & $8(4)$ & $9(5)$ & 0 & 0 & 0 & 0 & 0.22 \\
\hline Poeciliidae & 4. Poecilia reticulata & $4(5)$ & 0 & $6(5)$ & $10(4)$ & 0 & 0 & 0 & 0 & 0.03 \\
\hline Heptapteridae & 5. Rhamdia quelen & 0 & 0 & 0 & 0 & 0 & $1(8)$ & $1(7)$ & 0 & -0.22 \\
\hline Loricariidae & 6. Chaetostoma dorsale & $511(1)$ & $532(1)$ & $463(1)$ & $318(1)$ & $62(2)$ & $53(3)$ & $82(3)$ & $16(3)$ & $0.94 * *$ \\
\hline Loricariidae & 7. Chaetostoma milesi & 0 & 0 & 0 & 0 & $81(1)$ & $98(2)$ & $174(1)$ & $37(2)$ & $-0.73^{* *}$ \\
\hline Loricariidae & 8. Farlowella acus & 0 & 0 & 0 & 0 & $3(7)$ & $4(7)$ & $1(7)$ & 0 & $-0.54 * *$ \\
\hline Loricariidae & 9. Hypostomus plecostomoides & 0 & 0 & 0 & 0 & $2(8)$ & 0 & $1(7)$ & 0 & $-0.46^{*}$ \\
\hline Pseudopimelodidae & 10. Batrochoglanis raninus & 0 & 0 & 0 & 0 & $1(9)$ & 0 & $2(6)$ & 0 & $-0.46^{*}$ \\
\hline Trichomycteridae & 11. Trichomycterus cf arleoi & $34(3)$ & $28(3)$ & 19(3) & $19(3)$ & $24(4)$ & $30(4)$ & $8(5)$ & $4(6)$ & $0.77 * *$ \\
\hline Trichomycteridae & 12. Trichomycterus sp. "Turbio" & $4(5)$ & 0 & $4(6)$ & $1(6)$ & $12(6)$ & $17(5)$ & $21(4)$ & $15(4)$ & $-0.46^{*}$ \\
\hline
\end{tabular}

Números de colección para cada especie en CPUCLA: 1(3 525), 2(3 526), 3(3 513), 4(3 505), 5(3 529), 6(3 501), 7(3 514), $8(3527), 9(3534), 10(3535), 11(3502)$ y 12(3 521). $(\mathrm{p}<0.05=*, \mathrm{p}<0.01=* *)$.

Keough, 2002). Para evaluar la estabilidad en las comunidades de peces durante el periodo, se exploró la variación en la abundancia y en sus rangos para cada especie; además, la estabilidad se interpretó mediante coeficientes de variación $[\mathrm{CV}=$ desviación estándar/promedio X 100] (Grossman et al., 1990).

Para identificar grupos de ictiofaunas se elaboró un análisis de clasificación jerárquica con base en la distancia Euclídea y enlace Ward (Legendre \& Legendre, 1998) y su ajuste se probó con una correlación cofenética. Para contrastar los grupos detectados y reconocer gradientes entre las localidades se desarrolló una ordenación de escalamiento multidimensional no métrico (NMS, McCune $\&$ Mefford, 2011). La abundancia se transformó $\left[\log _{10}(\mathrm{x}+1)\right] \mathrm{y}$ todas las especies fueron incluidas. Se usó la distancia Euclídea y el estrés fue evaluado usando una prueba de Monte Carlo (250 permutaciones, $\mathrm{p}=0.004$ ). Las tendencias dentro de las localidades fueron reconocidas con vectores de sucesión. Se exploraron gradientes superponiendo las variables ambientales sobre los ejes de la ordenación y su relación se examinó con coeficientes de correlación de Spearman. Se aplicó un procedimiento no paramétrico de permutación de respuestas múltiples (MRPP, distancia Euclídea) para probar las diferencias entre los grupos detectados en los análisis multivariados. El MRPP no requiere de supuestos normalidad multivariada y homogeneidad de varianzas y prueba las diferencias entre la estructura de las comunidades entre grupos mediante un valor del estadístico $T$, el cual describe la separación entre grupos: la separación es más fuerte según aumenten los valores negativos del estadístico $T$ (McCune \& Mefford, 2011).

Para evaluar el estado de conservación de las localidades se empleó el índice de integridad de hábitat (HII, Nessimian et al., 2008), compuesto de doce medidas que evalúan la integridad del río según el estado de la vegetación ribereña y uso de la tierra, la geomorfología y características del cauce, la vegetación acuática y los detritos. Aquí el HII varió entre 0 y 100 y se interpretó como el estado de conservación 
de la localidad de muestreo. La asociación de las variables ambientales y los atributos de los peces respecto a la altura y al HII se evaluó con correlaciones de Spearman. Los análisis multivariados se elaboraron con PC-ORD $6(\mathrm{McCu}-$ ne \& Mefford, 2011) y el resto con PAST 3.0 (Hammer, Harper, \& Ryan, 2001).

\section{RESULTADOS}

Al disminuir la altura se incrementó la cobertura de arena en el sustrato (Spearman, $\left.\mathrm{r}_{\mathrm{s}}=0.51, \mathrm{p}=0.0112\right)$ y en las aguas aumentó la temperatura $\left(\mathrm{r}_{\mathrm{s}}=-0.81, \mathrm{p}<0.0001\right.$, intervalo 18.1$\left.28.1^{\circ} \mathrm{C}\right)$, la conductividad $\left(\mathrm{r}_{\mathrm{s}}=-0.77, \mathrm{p}<0.0001\right.$, $280-1010 \mu \mathrm{S} / \mathrm{cm}$ ) y los sólidos disueltos totales $\left(r_{s}=-0.77, p<0.0001,145-474 \mathrm{mg} / \mathrm{L}\right)$. Entre las localidades de Río Claro, hubo diferencias entre las coberturas de sombra (Kruskal-Wallis, $\mathrm{H}=6.62, \mathrm{p}=0.0366$ ), conductividad y sólidos disueltos totales (ambas: $\mathrm{H}=9.85, \mathrm{p}=0.00728$ ) y temperatura $(\mathrm{H}=7.34, \mathrm{p}=0.02519)$. Resultados similares fueron detectados entre las localidades del Turbio (pruebas no presentadas), salvo por las diferencias de la cobertura de arena entre las mismas $(\mathrm{H}=6.038, \mathrm{p}=0.0488)$. En Río Claro la cobertura de rocas, (Mann-Whitney, $\mathrm{U}=5, \mathrm{p}=0.0001)$, ramas y hojas $(\mathrm{U}=16.5$, $\mathrm{p}=0.0015)$ y sombra $(\mathrm{U}=34, \mathrm{p}=0.0300)$ fueron significativamente mayores respecto al Turbio, pero en este último fueron mayores las coberturas de gravas finas $(U=31, p=0.0194)$ y gruesas $(\mathrm{U}=16, \mathrm{p}=0.0014)$, así como la conductividad $(\mathrm{U}=27, \mathrm{p}=0.0102)$ y sólidos disueltos totales $(\mathrm{U}=28, \mathrm{p}=0.0120)$, sugiriendo diferencias del estado de conservación entre los tributarios. Las coberturas del sustrato no fueron diferentes entre el inicio de la sequía (enero) y el inicio de las lluvias (abril) en los dos tributarios (Wilcoxon, todas las localidades $\mathrm{p}>0.1$ ). En el mismo periodo aumentó la conductividad, los sólidos disueltos totales y la temperatura pero sin significancia estadística.

Entre las 12 especies capturadas (Cuadro 2) predominaron los Siluriformes (8 spp.) y los Characiformes (3 spp.). La riqueza disminuyó con el incremento de la altura $\left(\mathrm{r}_{\mathrm{s}}=-0.69\right.$, $\mathrm{p}=0.0001$ ), con ocho especies a los $770 \mathrm{msnm}$ y tres (Creagrutus taphorni Vari \& Harold 2001, Chaetostoma dorsale Eigenmann 1922 y Trichomycterus cf arleoi (Fernández-Yépez 1972)) a partir de los 1 100msnm. C. taphorni se registró en todas las localidades, $C$. dorsale no se encontró por debajo de los $900 \mathrm{msnm}$, y Bryconamericus cismontanus Eigenmann 1914, Chaetostoma milesi Fowler 1941 y Farlowella acus (Kner 1853) no sobrepasaron esa altitud. Hypostomus plecostomoides (Eigenmann 1922) y Batrochoglanis raninus (Valenciennes 1840) solo se encontraron en los $770 \mathrm{msnm}$. La riqueza no fue diferente entre enero y abril, ya sea en Río Claro (Wilcoxon, $\mathrm{W}=3, \mathrm{p}=0.1573$ ) o en el Turbio ( $\mathrm{W}=3, \mathrm{p}=0.1797$ ).

Se registraron 3774 individuos, donde los Siluriformes acumularon el $71.1 \%$ de la abundancia (Loricariidae: 64.6\%) y los Characiformes el $28.4 \%$. La localidad RC1 contuvo la mayor abundancia entre todas las localidades $(48.6 \%)$ y lo contrario ocurrió en RC2 (0.98\%), mientras que en el Turbio las abundancias fueron bajas (Fig. 1, Cuadro 2). La abundancia incrementó poco con la altura $\left(\mathrm{r}_{\mathrm{s}}=0.35, \mathrm{p}=0.0946\right)$, siendo regida por $C$. dorsale $(53.9 \%)$, C. taphorni $(26.5 \%)$ y C. milesi (10.3\%). Las especies con mayores rangos de abundancia se mantuvieron en el tiempo: en Río Claro y Guayamure; C. dorsale, C. taphor$n i$ y Trichomycterus of arleoi mantuvieron rangos invariables (Cuadro 2), pero en el Turbio los primeros rangos se repartieron entre $C$. milesi y C. taphorni. En la mayoría de las localidades (Fig. 1) la abundancia tuvo un máximo en marzo y una disminución en abril. En Río Claro la disminución de la abundancia varió menos entre marzo y abril (intervalo 19-38\%) en comparación con el Turbio (intervalo 50-76\%); sin embargo, la abundancia no varió entre enero y abril para Río Claro (Wilcoxon, $\mathrm{W}=5, \mathrm{p}=0.2850)$ y el Turbio $(\mathrm{W}=6, \mathrm{p}=0.1088)$. La especies dominantes tuvieron coeficientes de variación (CV) amplios, como C. taphorni (22.4-89\%) y C. dorsale (23.7-115.5\%), ambas con valores extremos en el Río Claro. En RC2 la abundancia fue muy baja y con $\mathrm{CV}$ elevados (89-116\%), así como la mayoría de las especies en localidades a menor altura en el Turbio. 

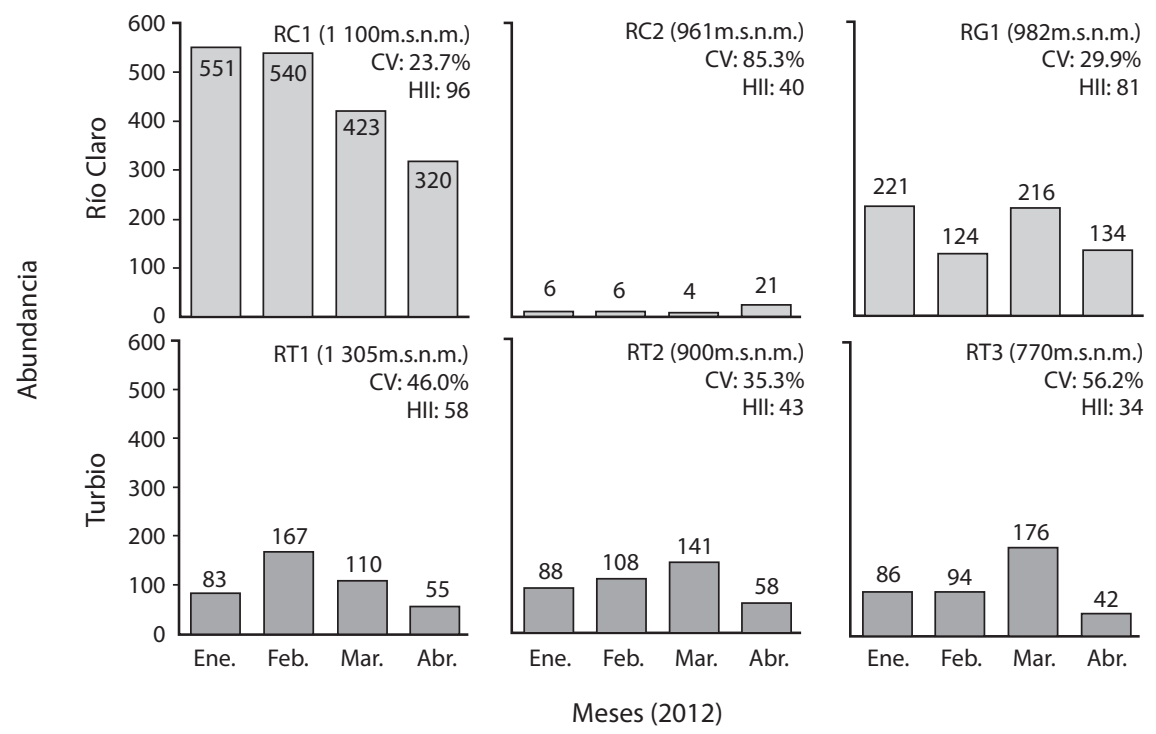

Fig. 1. Variación mensual en la abundancia de la ictiofauna. Los coeficientes de variación (CV) y el HII son únicos para el periodo. Los gráficos tienen igual escala vertical.

Fig. 1. Monthly variation in abundance of ichthyofauna. The coefficients of variation (CV) and HII are unique for the period. The graphs have the same vertical scale.

Los CV fueron menores a mayores alturas, con excepción de la localidad RC2 (Fig. 1). Lo anterior sugiere que las comunidades de peces fueron moderadamente estables durante el periodo evaluado, con valores entre el $46 \%$ y $56 \%$ en sus CV; además, la estabilidad decreció a menores alturas y también con un menor estado de conservación de las localidades.

En la clasificación multivariada (correlación cofenética, $\mathrm{r}=0.82$ ) las muestras en cada localidad tuvieron un arreglo generalmente consecutivo (Fig. 2a) y se detectaron grupos de localidades relacionadas con la altura y el estado de conservación de los ríos: grupo a ( $\mathrm{RC} 1, \mathrm{RG} 1$ y RT1), grupo b (RT2 y RT3) y grupo c (RC2). El NMS tuvo una solución bidimensional, una varianza acumulada muy elevada (96.1\%) y bajo estrés medio (7.58). El primer eje explicó en gran medida la varianza en las comunidades de peces (NMS1: 77.3\%; NMS2: 18.8\%; Fig. 2b) y se utilizó para análisis posteriores. Los vectores de sucesión indicaron que las comunidades cambiaron muy poco durante el periodo. En el NMS se detectaron los mismos grupos que en la clasificación multivariada y estos fueron significativamente diferentes (MRPP, grupos a-b: $\mathrm{T}=-5.86, \mathrm{~A}=0.215, \mathrm{p}=0.0011$; grupos a-c: $\mathrm{T}=-6.48, \mathrm{~A}=0.445, \mathrm{p}=0.0003$; grupos b-c: $\mathrm{T}=-4.73, \mathrm{~A}=0.247, \mathrm{p}=0.0013)$.

Las variables ambientales cambiaron a lo largo de un gradiente resumido por el eje NMS1 (Cuadro 1) y expresado por mayores valores en las coberturas de sombra, rocas y de ramas y hojas, así como por el incremento en la temperatura, conductividad, sólidos disueltos totales, el ancho del cauce y la velocidad de la corriente (Fig. 2b). El hábitat varió entre los grupos a-b $(\mathrm{T}=-8.23, \mathrm{~A}=0.280, \mathrm{p}<0.0001)$ y los grupos a-c $(\mathrm{T}=-3.82, \mathrm{~A}=0.151, \mathrm{p}=0.0055)$; esto es, localidades a mayores alturas (RC1, RG1, RT1) tuvieron diferencias en sus hábitats respecto a las de menor altura (RT2, RT3), y con menor estado de conservación (RC2). Los grupos (b-c) no fueron diferentes en sus hábitats (MRPP, $\mathrm{T}=0.99, \mathrm{~A}=-0.057, \mathrm{p}=0.8721$ ).

El estado de conservación (HII) fue en promedio mayor en Río Claro respecto al Turbio, así como también entre los grupos de localidades (Cuadro 1, Fig. 1). Mayores valores del HII se asociaron con mayores coberturas 
A

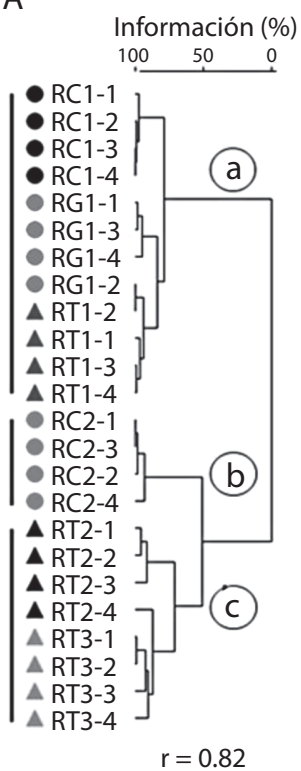

B

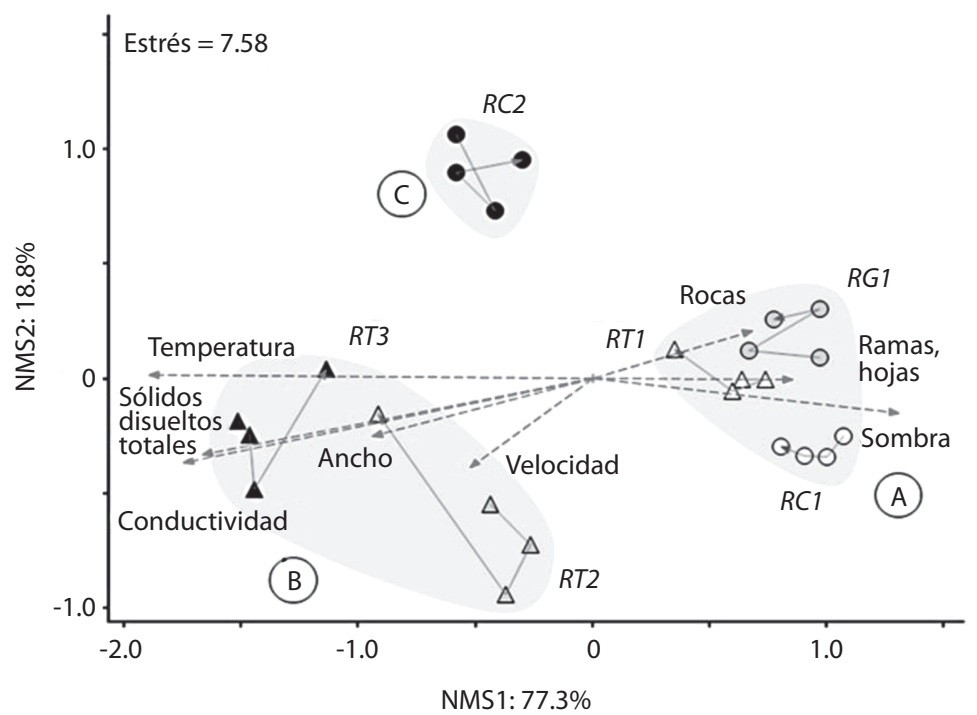

Fig. 2. Análisis de clasificación jerárquica (a): para cada localidad la secuencia es enero $=1$, febrero $=2$, marzo $=3$, abril $=4$. Se indican los grupos de localidades (a, b, c). Ordenación NMS (b): las líneas conectando muestras denotan la secuencia temporal, las variables ambientales se indican con línea punteadas y las áreas sombreadas muestran los grupos (a, b, c).

Fig. 2. Hierarchical classification analysis (a): for each site the sequence is January=1, February=2, March=3, April=4. Groups of sites are indicated ( $a, b, c)$. Ordination NMS (b): the lines connecting samples denoted the temporal sequence, environmental variables are indicated by dotted lines and the shaded areas show the groups (a, b, c).

de sombra $\left(\mathrm{r}_{\mathrm{s}}=0.72, \mathrm{p}<0.0001\right.$, Fig. 3a), así como de ramas y hojas $\left(\mathrm{r}_{\mathrm{s}}=0.59, \mathrm{p}=0.0026\right.$, Fig. $3 b)$ y rocas $\left(r_{s}=0.4934, p=0.0142\right)$ en los cauces. Al contrario, menores valores del HII se correlacionaron con mayores coberturas de grava gruesa $\left(\mathrm{r}_{\mathrm{s}}=-0.53, \mathrm{p}=0.0074\right)$ y cantos rodados $\left(\mathrm{r}_{\mathrm{s}}=-0.46, \mathrm{p}=0.0236\right)$ en los cauces, así como con la elevación de la temperatura $\left(r_{\mathrm{s}}=-0.76, \mathrm{p}<0.0001\right)$, la conductividad $\left(\mathrm{r}_{\mathrm{s}}=-\right.$ $0.63, \mathrm{p}=0.0010)$ y la concentración de sólidos disueltos totales $\left(\mathrm{r}_{\mathrm{s}}=-0.62, \mathrm{p}=0.0012\right)$ en las aguas (Fig. 3a, 3b). El HII tuvo asociación alta con la riqueza de especies $\left(r_{s}=-0.75, p<0.0001\right)$ pero irrelevante con la abundancia $\left(r_{s}=0.11\right.$, $\mathrm{p}=0.6228$ ). La mayor correlación del HII fue con el eje NMS1 $\left(r_{s}=0.95, p<0.0001\right)$ y los coeficientes de variación $\left(\mathrm{r}_{\mathrm{s}}=-0.89, \mathrm{p}=0.0167\right.$; Fig. $3 \mathrm{c}$ ). Todas las asociaciones indicaron que un mayor estado de conservación se asoció con cauces más heterogéneos, comunidades de peces menos fluctuantes en el espacio multivariado y, a su vez, con una mayor estabilidad.

\section{DISCUSIÓN}

La riqueza y abundancia de especies no cambió significativamente durante el periodo evaluado, pero sí en cuanto a la altura y el estado de conservación de los ríos, como es reconocido en montañas andinas en cuanto a la disminución de la riqueza y la dominancia de pocas especies a mayores alturas (JaramilloVilla et al., 2010) o por la disrupción de estos patrones por efecto de la intervención humana (Lujan et al., 2013). La riqueza de especies fue mucho menor a la reportada en cuencas contiguas que drenan al río Orinoco (Acarigua) o al mar Caribe (Tocuyo), en donde unas 30 especies habitan en la transición entre los piedemontes y las montañas (300-800msnm) 

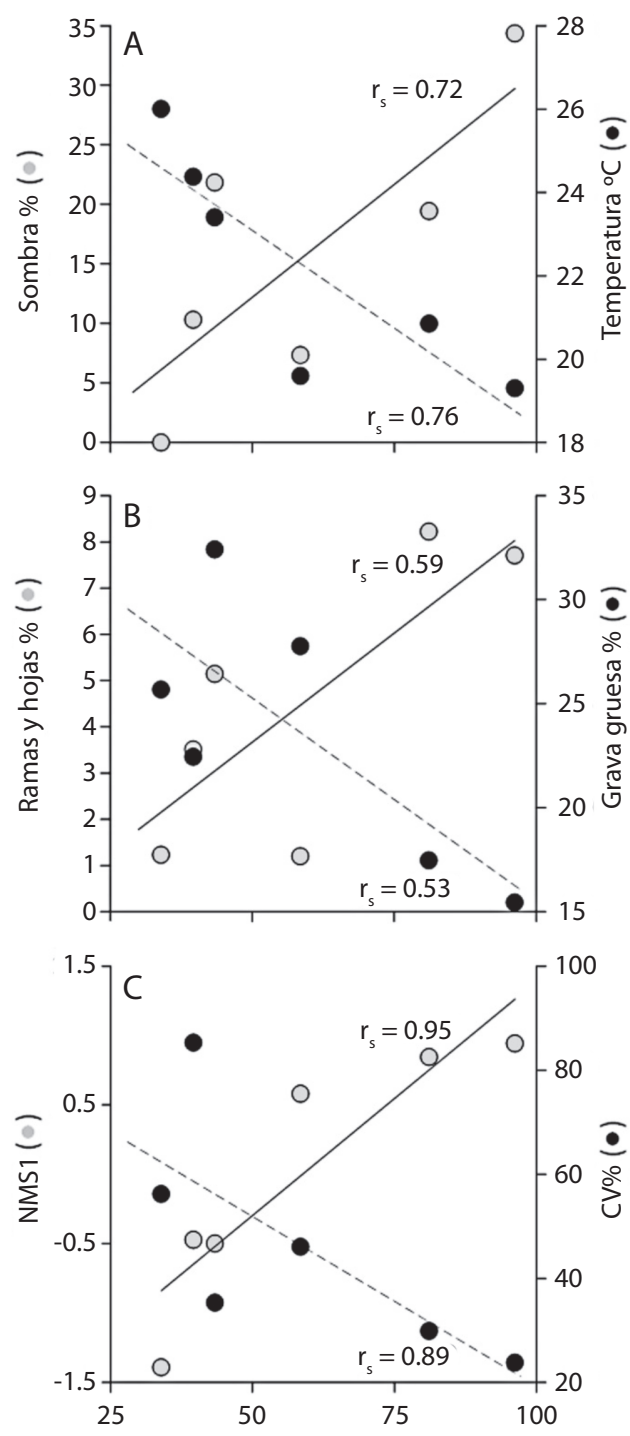

Índice de integridad de hábitat

Fig. 3. Relación del índice de integridad de hábitat (HII) con (a) la cobertura de sombra y la temperatura, (b) con coberturas de troncos, ramas y hojas, y grava gruesa, y (c) con el eje NMS1 y los coeficientes de variación (CV). Fig. 3. Relation of habitat integrity index (HII) with (a) cover of shade and temperature, (b) cover of logs, branches and leaves, and coarse gravel, and (c) with the axis NMS1 and coefficients of variation $(\mathrm{CV})$.

(Allan et al., 2006; Rodríguez-Olarte et al., 2006). Lo anterior sugiere que en la cuenca alta del río Turbio las comunidades posiblemente son residuales o simplificadas como respuesta a la combinación del clima semiárido regional y la intervención humana; estas comunidades estarían compuestas principalmente por poblaciones remanentes, pues la intermitencia de los caudales, la elevada contaminación de las aguas y la pérdida generalizada del hábitat acuático sugieren una colonización limitada.

Las ictiofaunas fueron predecibles $\mathrm{y}$ moderadamente estables durante el período evaluado. En los piedemontes andinos del Orinoco las comunidades de peces varían menos que las de los llanos altos inmediatos; además, sus cambios pueden ser predecibles en un ciclo anual y hasta en un periodo climático, como sequía o lluvias (Winemiller et al., 2008). La suma de estos resultados sugiere que la estabilidad de las comunidades de peces aumenta desde los llanos hasta las montañas. Este gradiente en la estabilidad tiene relación con una menor variabilidad ambiental en los ríos de montaña (Grossman et al., 1990), donde existe una menor complejidad del hábitat fluvial y una menor productividad en las aguas respecto a corrientes a menor altura o en las planicies; además, algunos atributos del hábitat son determinantes en la estabilidad, como la variación del caudal (Grossman, Ratajczak, Farr, Wagner, \& Petty, 2010), lo que también puede ser una expresión del estado de conservación de los ríos. Los ríos seccionados durante la sequía, con sustratos homogéneos y/o carentes de sombra limitan la colonización y permanencia de los peces, pero a su vez pueden contener comunidades más simples y tolerantes que las previstas en condiciones naturales y mostrar elevada estabilidad en el tiempo.

En el área de estudio, la intervención humana es la causa fundamental en la homogeneidad de los sustratos y la pérdida de sombra, así como el aumento en los sólidos disueltos totales y la temperatura, lo que compromete la conservación de la ictiofauna; además, los parques nacionales locales contienen una pequeña fracción de la ictiofauna y más allá de sus fronteras los tributarios se encuentran muy intervenidos, una situación de alcance regional (Rodríguez-Olarte, Taphorn \& Lobón-Cerviá, 
2011) y que probablemente ocurre en el resto de los Andes. Por otro lado, las previsiones del cambio climático indican una disminución de las precipitaciones y un aumento de la temperatura (Ovalles, Cortéz, Rodríguez, Rey, \& Cabrera-Bisbal, 2008), lo cual puede ser perjudicial para los peces en las montañas andinas, ya que pueden ser muy sensitivos debido a sus franjas de distribución restringida (Lujan et al., 2013). Durante el periodo de estudio Río Claro se secó completamente por debajo de los $800 \mathrm{msnm}$, para resurgir en las planicies, mientras que el Turbio fue intermitente al ingresar a las mismas pero sólo al final de la sequía. Se estima que los impactos tendrán una intensidad mayor sobre la ictiofauna local al considerar los escenarios del cambio climático, pues en sequía se fragmentarían aún más los ríos, con la retracción de las comunidades de peces remanentes y, en el peor de los casos, la extirpación de poblaciones locales con características únicas (e.g. Trichomycterus). En consecuencia, los tributarios y los peces en la cuenca alta del río Turbio deben ser considerados en situación de riesgo y con prioridad de conservación.

\section{AGRADECIMIENTOS}

Este trabajo es un resultado parcial del proyecto 002-AG-2012 (CDCHT-UCLA). INSOPESCA-MPPAT otorgó los permisos de pesca. En los muestreos colaboraron Héctor Rivera y estudiantes de la UCLA. Agradecemos por la revisión del manuscrito a Donald C. Taphorn y los árbitros anónimos.

\section{RESUMEN}

La mayoría de los ríos en las montañas andinas de Venezuela en la cuenca del Orinoco sufren variadas perturbaciones. A pesar de esto, sus comunidades de peces han sido poco evaluadas en términos de sus gradientes espaciales y temporales. La generación de información sobre estas comunidades es prioritaria, y puede impulsar la conservación de los recursos hidrobiológicos regionales. Con este fin, seis localidades fueron evaluadas mensualmente en tributarios de la cuenca alta del río Turbio en la Sierra de Portuguesa (770-1305msnm) durante el período de sequía y el inicio del período de lluvias (enero-abril de 2012), se caracterizaron las dimensiones del cauce, el sustrato y las variables físico-químicas del agua. Se utilizó electropesca para determinar el número y abundancia de especies de peces. Las comunidades de peces y su estabilidad se evaluaron explorando los cambios en la riqueza y abundancia y por coeficientes de variación, clasificación jerárquica y análisis de escalamiento multidimensional no métrico. El estado de conservación de las localidades se estimó con un índice de integridad del hábitat. La riqueza de 12 especies varió según la elevación, los tributarios y el estado de conservación de las localidades. Chaetostoma dorsale, Creagrutus taphorni y Chaetostoma milesi comprendieron el $90.8 \%$ de la abundancia relativa y mantuvieron los primeros rangos durante el período. Las comunidades tuvieron poca variación espacio-temporal y esta se expresó en un gradiente asociado principalmente por las coberturas de sombra, rocas y grava, la temperatura del agua y la conductividad. La estabilidad fue moderada, variando con la elevación y el estado de conservación de las localidades. Los tributarios en la cuenca alta del río Turbio se encuentran bajo impactos severos y sus ictiofaunas son empobrecidas y en situación de riesgo.

Palabras clave: comunidades de peces, gradientes ambientales, ríos andinos, recursos hidrobiológicos, Venezuela.

\section{REFERENCIAS}

Allan, J. D. (2004). Landscapes and riverscapes: the influence of land use on stream ecosystems. Annual Review of Ecology and Systematics, 35, 257-284.

Allan, J. D., Flecker, A. S., Segnini, S., Taphorn, D. C., Sokol, E., \& Kling, G. W. (2006). Limnology of Andean piedmont rivers of Venezuela. Journal of the North American Benthological Society, 25, 66-81.

Anderson, E. P., \& Maldonado-Ocampo, J. A. (2011). A regional perspective on the diversity and conservation of tropical Andean fishes. Conservation Biology, $25,30-39$.

Barbour, M., Gerritsen, J., Snyder, B., \& Stribling, J. (1999). Rapid bioassessment protocols for use in streams and wadeable rivers: Periphyton, benthic macroinvertebrates, and fish. (EPA 841-B-99-002). (Recuperado http://www.epa.gov/owow/monitoring/ rbp/wp61pdf/rbp.pdf).

Froese, R., \& D. Pauly. Editors. 2013. FishBase. Recuperado en Agosto, 2013 de http://www.fishbase.org/

Grossman, G. D., Dowd, J. F., \& Crawford, M. (1990). Assemblages stability in stream fishes: A review. Environmental Management, 14, 661-671.

Grossman, G. D., Ratajczak, R. E., Farr, M. D., Wagner, C. M., \& Petty, J. T. (2010). Why there are fewer fish upstream. In K. B. Gido \& D. A. Jackson (Eds.), Community Ecology of Stream Fishes: Concepts, 
Approaches, and Techniques (pp. 63-82). Bethesda, MD: American Fisheries Society.

Hammer, Ø., Harper, D. A. T., \& Ryan, P. D. (2001). PAST.

Paleontological statistics software package for education and data analysis. Palaeontologica Electronica, 4, 9. (Recuperado de http://palaeo-electronica. org/2001_1/past/past.pdf).

Jaramillo-Villa, U., Maldonado-Ocampo, J. A., \& Escobar, F. (2010). Altitudinal variation in fish assemblage diversity in streams of the central Andes of Colombia, Journal of Fish Biology, 76, 2401-2417.

Legendre, P. \& Legendre. L. (1998). Numerical ecology. $2^{\text {nd }}$ English ed. Amsterdam: Elsevier Science BV.

Lujan, N. K., Roach, K. A., Jacobsen, D., Winemiller, K. O., Vargas, V. M., Ching, V. R., \& Maestre, J. A. (2013). Aquatic community structure across an Andes-to-Amazon fluvial gradient. Journal of Biogeography, 40, 1715-1728. (doi:10.1111/jbi.12131).

López, M. J. \& Andressen, R. (1996). Caracterización climática de las cuencas de los ríos Yacambú y Tocuyo en el ramal andino de la región centro occidental de Venezuela. Bioagro, 8, 87-95. Recuperado de http:// www.ucla.edu.ve/bioagro/Cont8-3.htm

McCune, B. \& Mefford, M. J. (2011). PC-ORD. Multivariate Analysis of Ecological Data. Version 6. Oregon: MjM Software.

Nessimian, J. L., Venticinque, E. M., Zuanon, J., De Marco, P. Jr., Gordo, M., Fidelis, L., Batista J. D., \& Juen, L. (2008). Land use, habitat integrity, and aquatic insect assemblages in Central Amazonian streams. Hydrobiologia, 614, 117-131.

Ovalles, F. A., Cortéz, A., Rodríguez, M. F., Rey, J. C., \& Cabrera-Bisbal, E. (2008). Variación geográfica en el impacto del cambio climático en el sector agrícola en Venezuela. Agronomía Tropical, 58, 37-40.

Quinn, G. P. \& Keough, M. J. (2002). Experimental design and data analysis for biologists. Cambridge: Cambridge University Press.

Rodríguez-Olarte, D., Coronel, J. L., Taphorn, D. C., \& Amaro, A. (2006). Los Peces del río Tocuyo, Vertiente del Caribe, Venezuela: un Análisis Preliminar para su conservación. Memoria de la Fundación La Salle de Ciencias Naturales, 165, 45-72. Recuperado de http://www2.scielo.org.ve/ scielo.php?script $=$ sci_arttext\&pid $=$ S0037-851820 $06000100002 \& \operatorname{lng}=$ pt\&nrm $=$ is

Rodríguez-Olarte, D., Taphorn, D. C., \& Lobón-Cerviá, J. (2011). Do protected areas conserve neotropical freshwater fishes? A case study of a biogeographic province in Venezuela. Animal Biodiversity and Conservation, 34, 273-285.

Smith, R. A. (1991). Ecología del Estado Lara. Edición especial $N^{\circ} 1$. Guanare: Biollania.

Winemiller, K. O., Agostinho A. A., \& Pellegrini-Caramaschi, E. (2008). Chapter 5: Fish ecology in tropical streams. In D. Dudgeon (Ed.), Tropical Stream Ecology (pp. 107-146). San Diego, CA: Elsevier Academic Press. 